
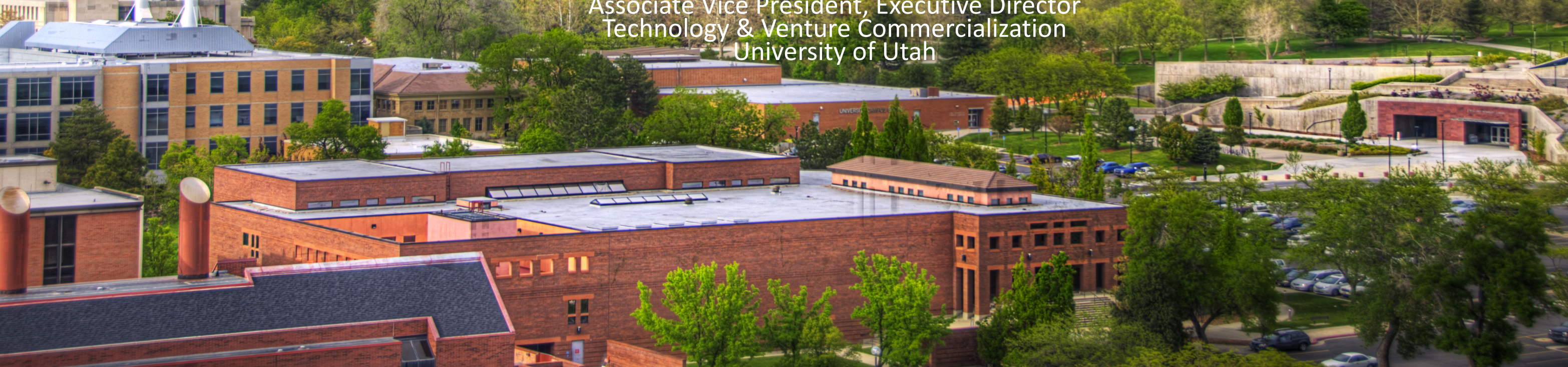

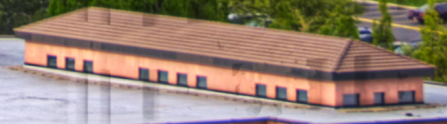
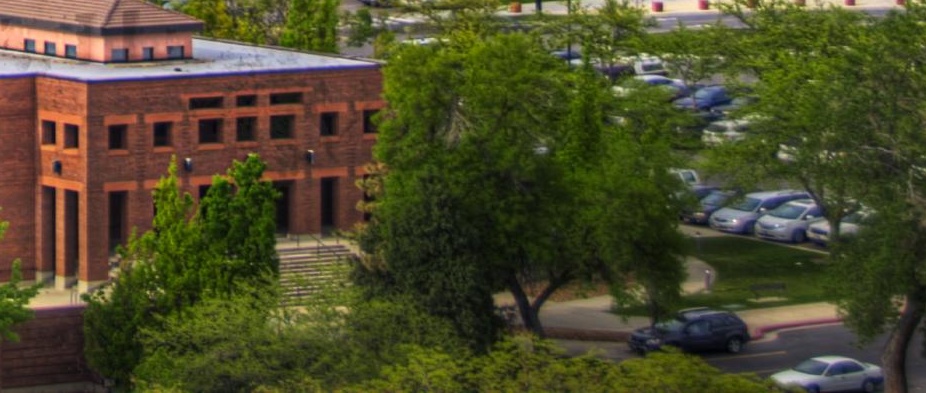
5
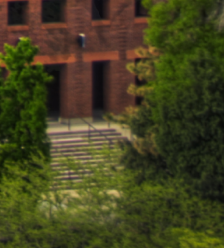


\section{Impact of commercialization}

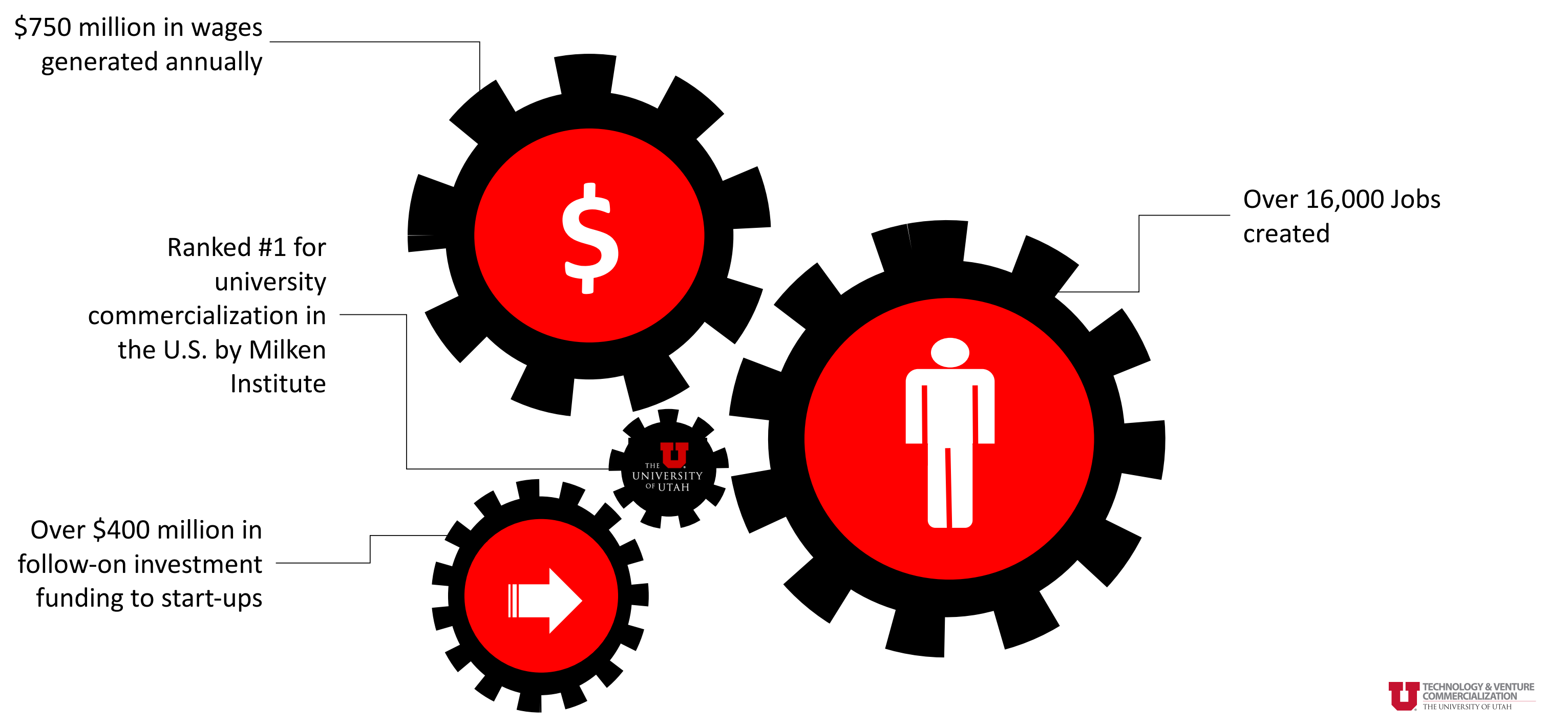




\section{Know what you bring to the ecosystem...}

Top 7 Disclosing Departments at University of Utah (2011-2018)

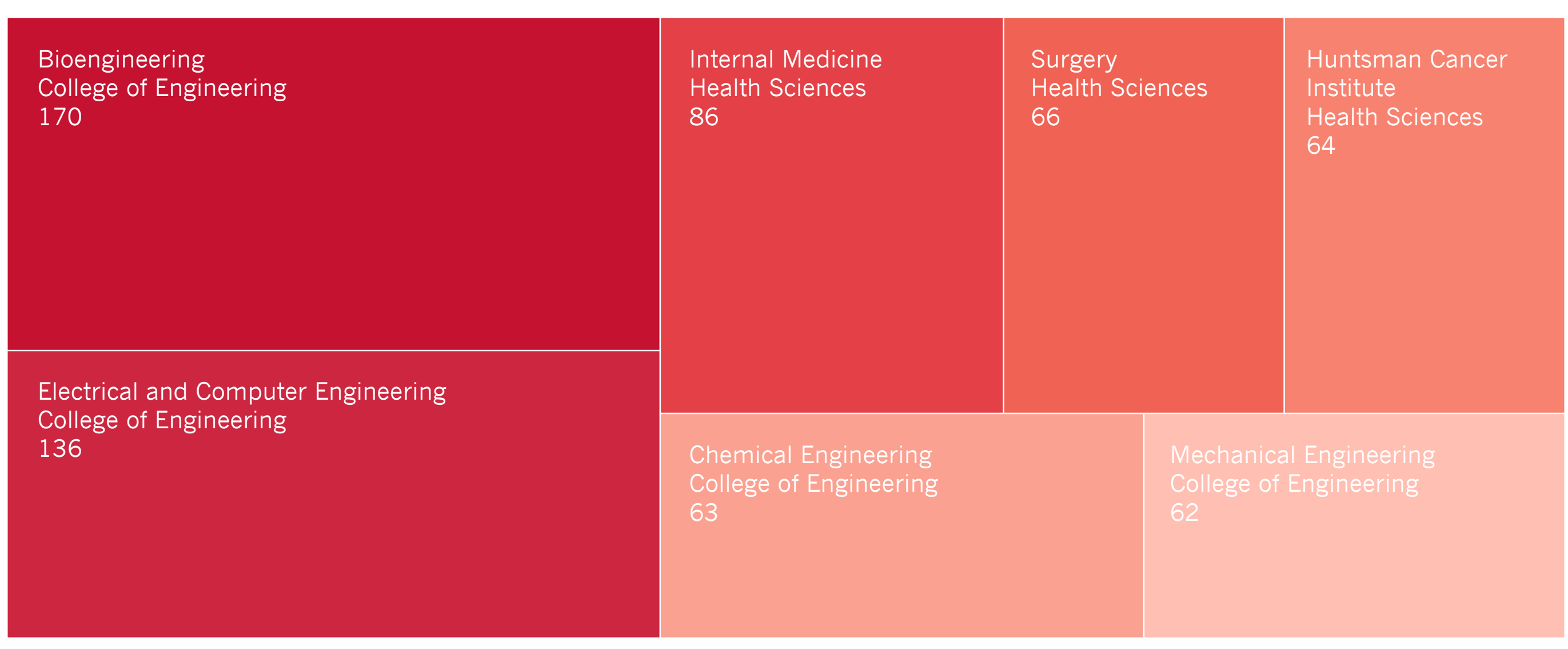




\section{Who brought you there...}

College of Engineering Disclosures at University of Utah (2011-2018)

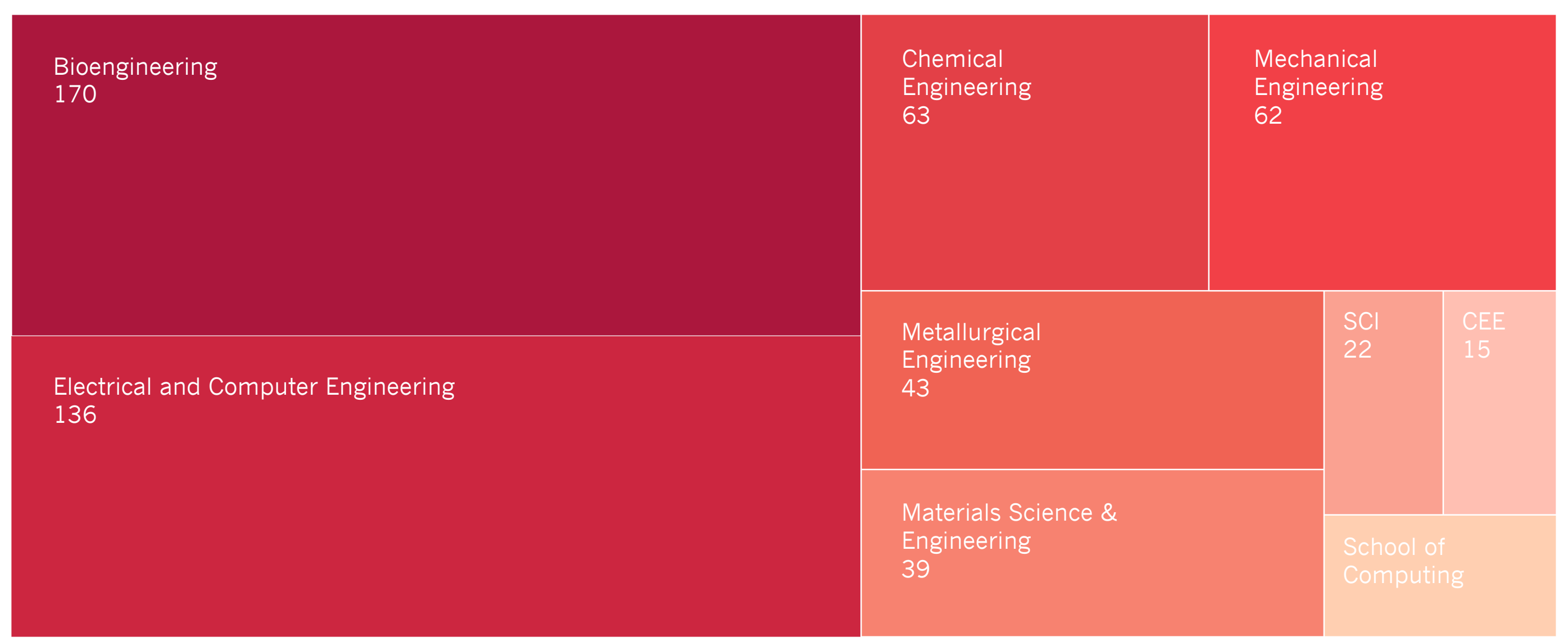




\section{What they want...}

College of Engineering Licenses at University of Utah (2011-2018)

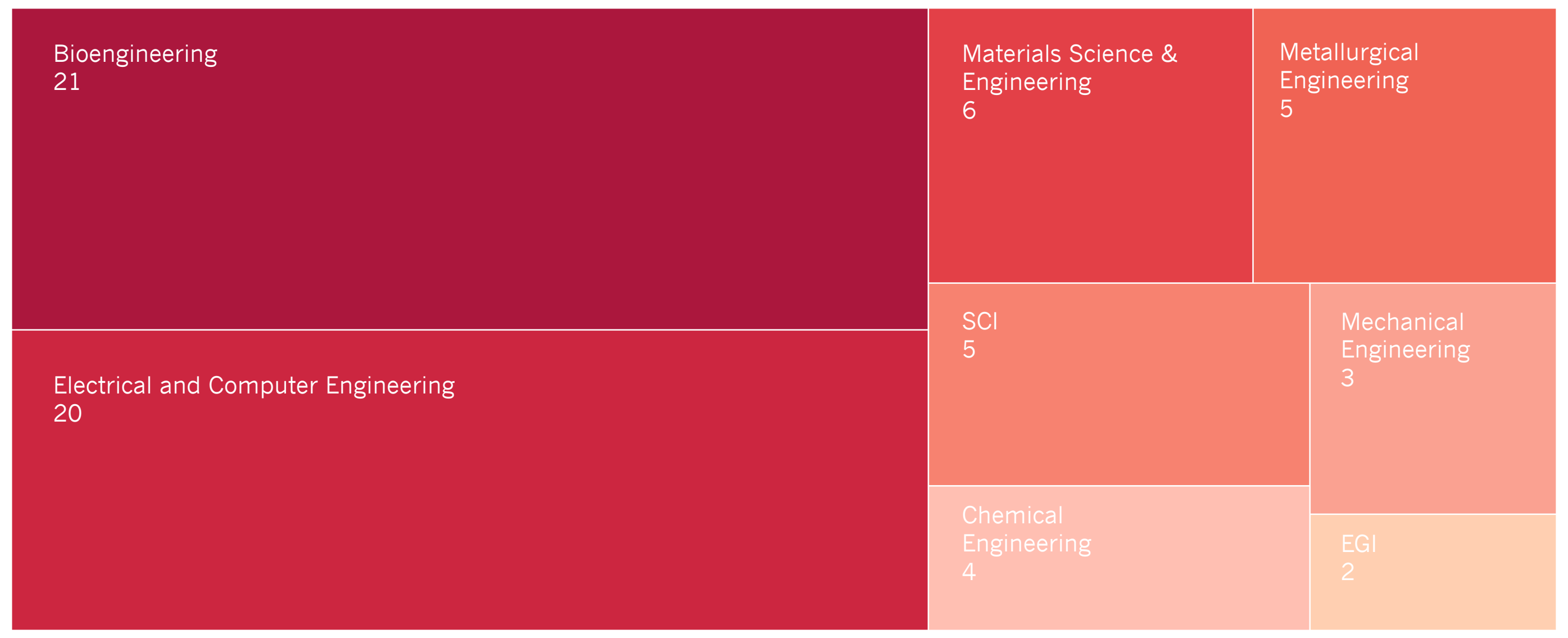




\section{What it takes to get there...}

College of Engineering Disclosures per $\$ 1$ million at University of Utah (2011-2018)

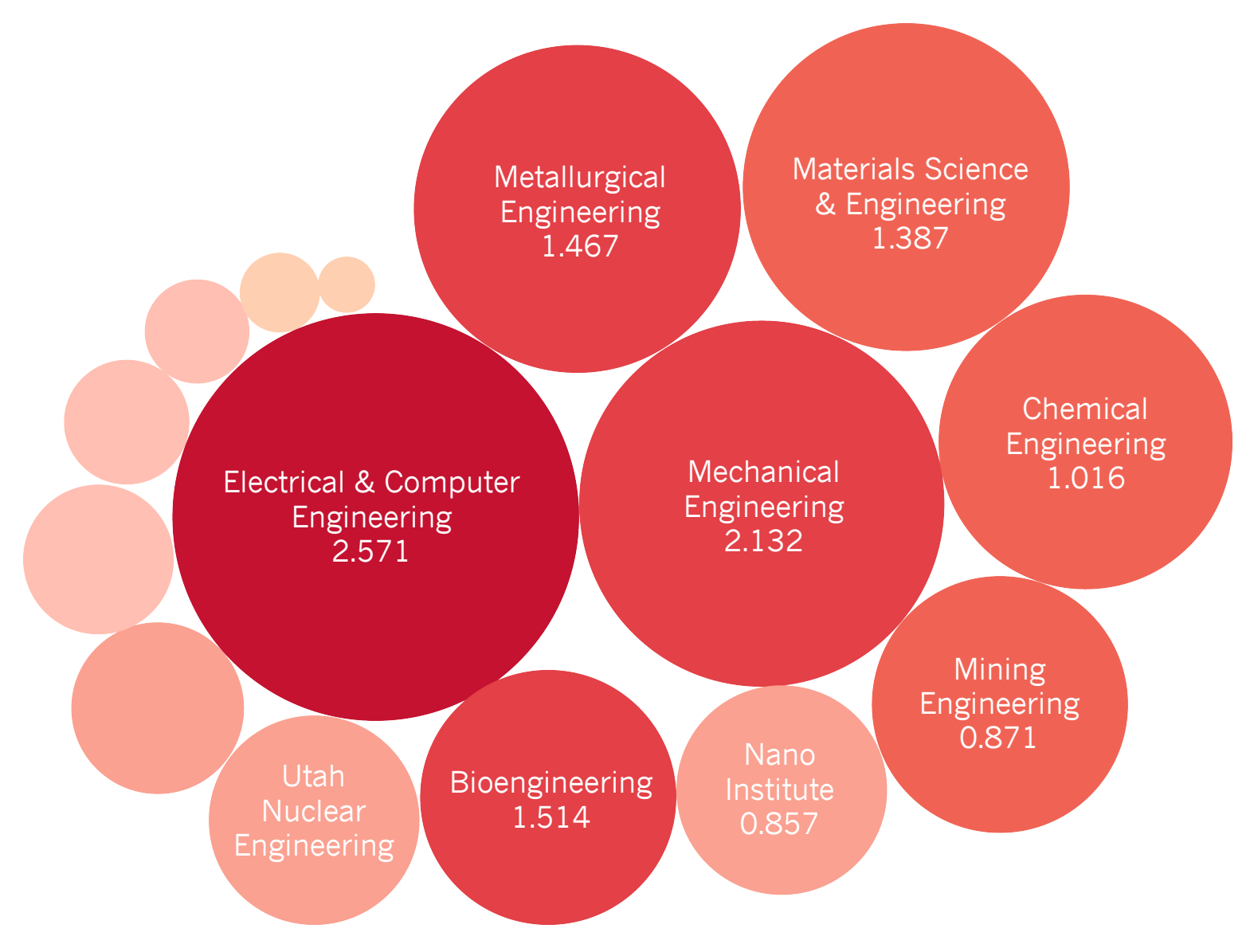




\section{And what you'll get out of it (and when)...}

Total Licensing Revenue at University of Utah
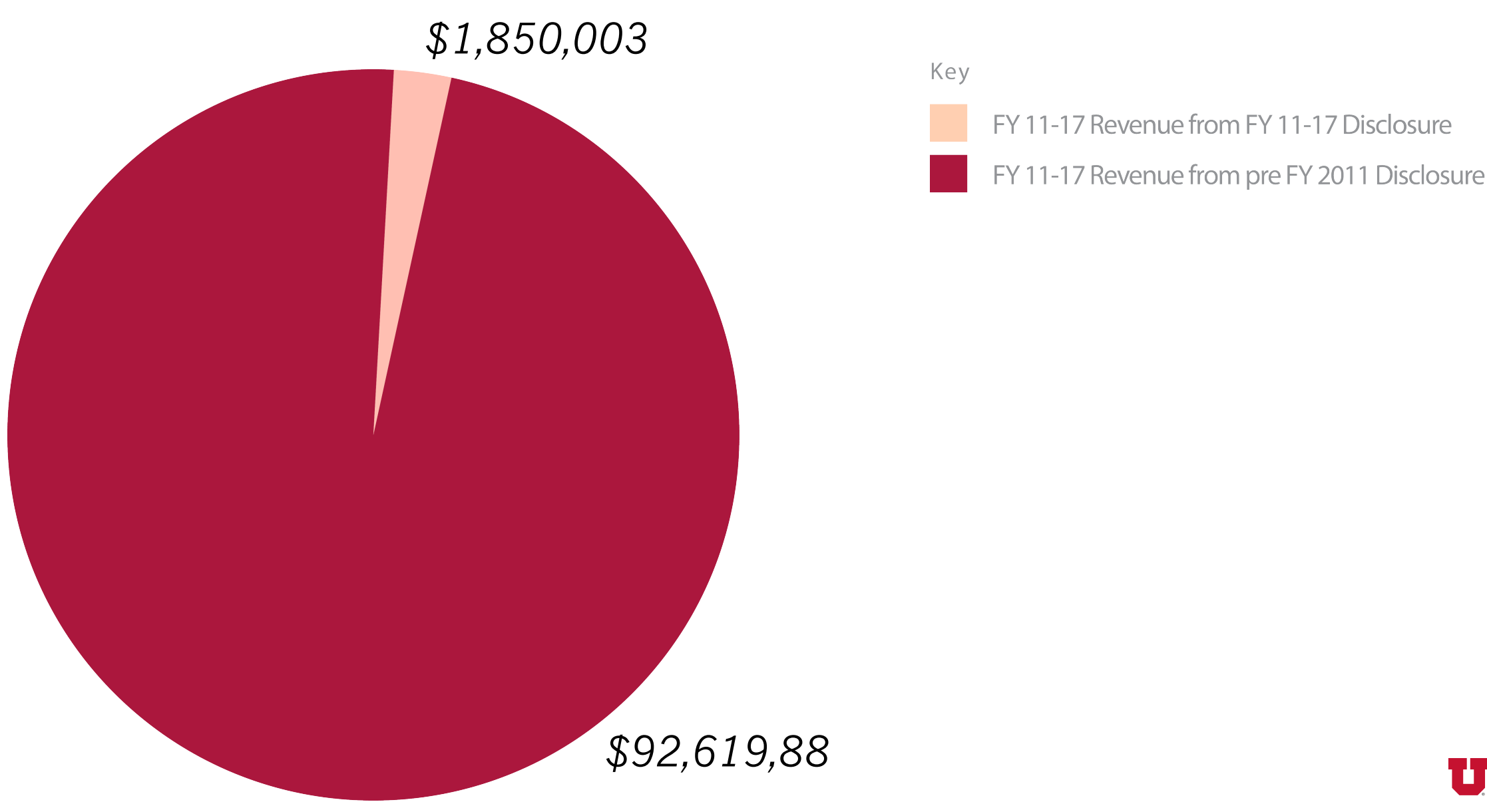


\section{Commercialization process}

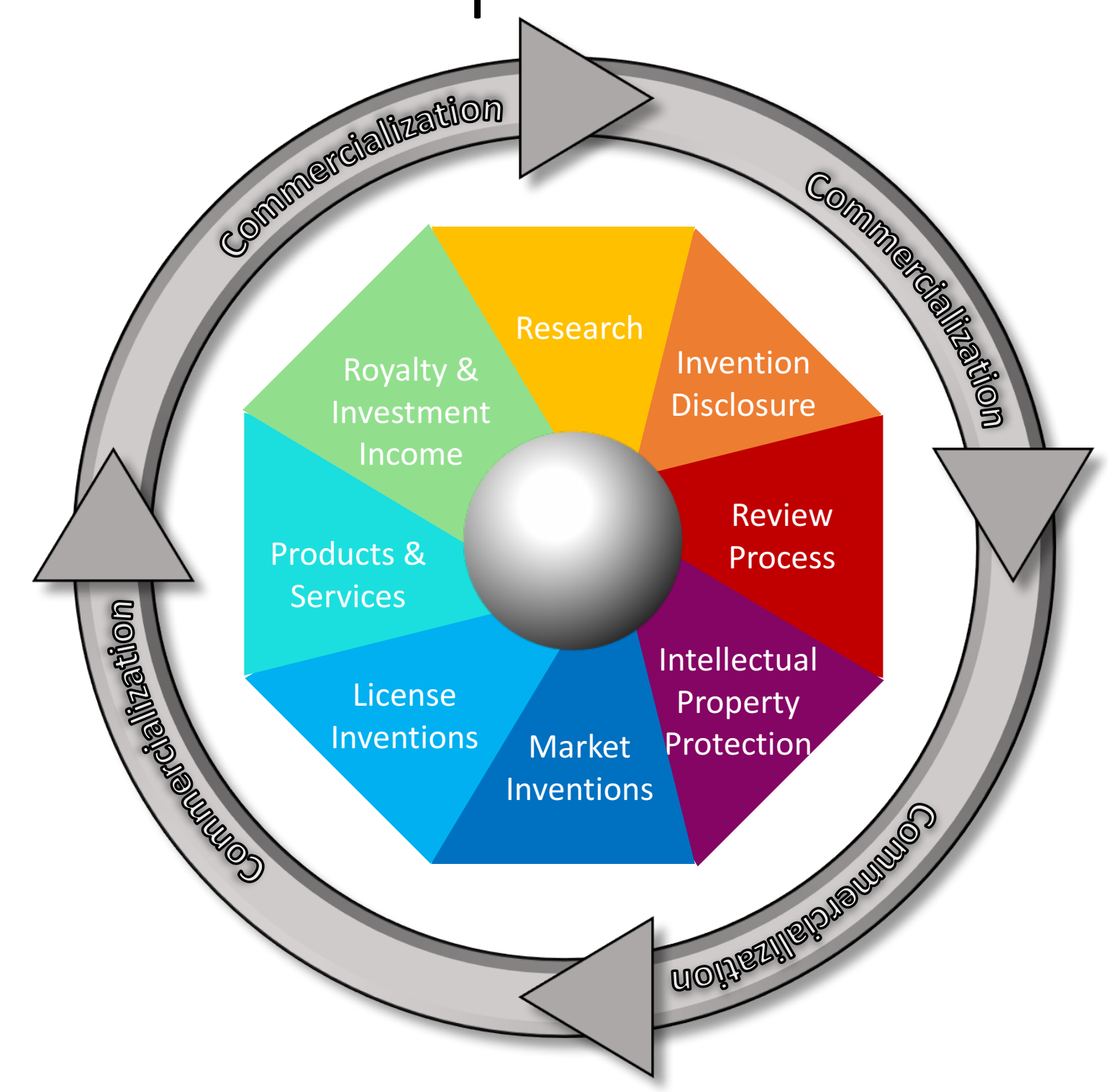




\section{Ecosystem realities}

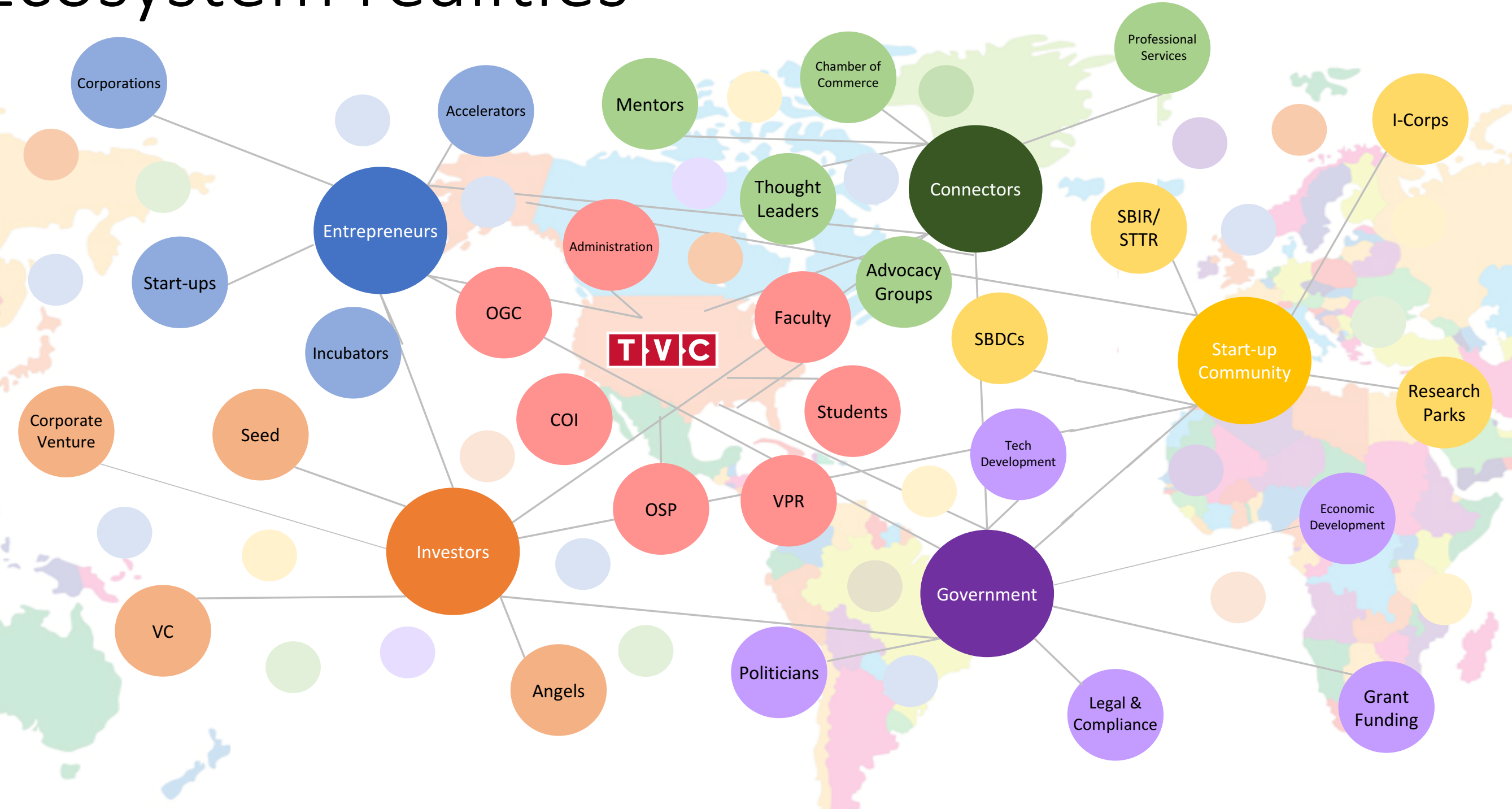


An ineffective innovation formula

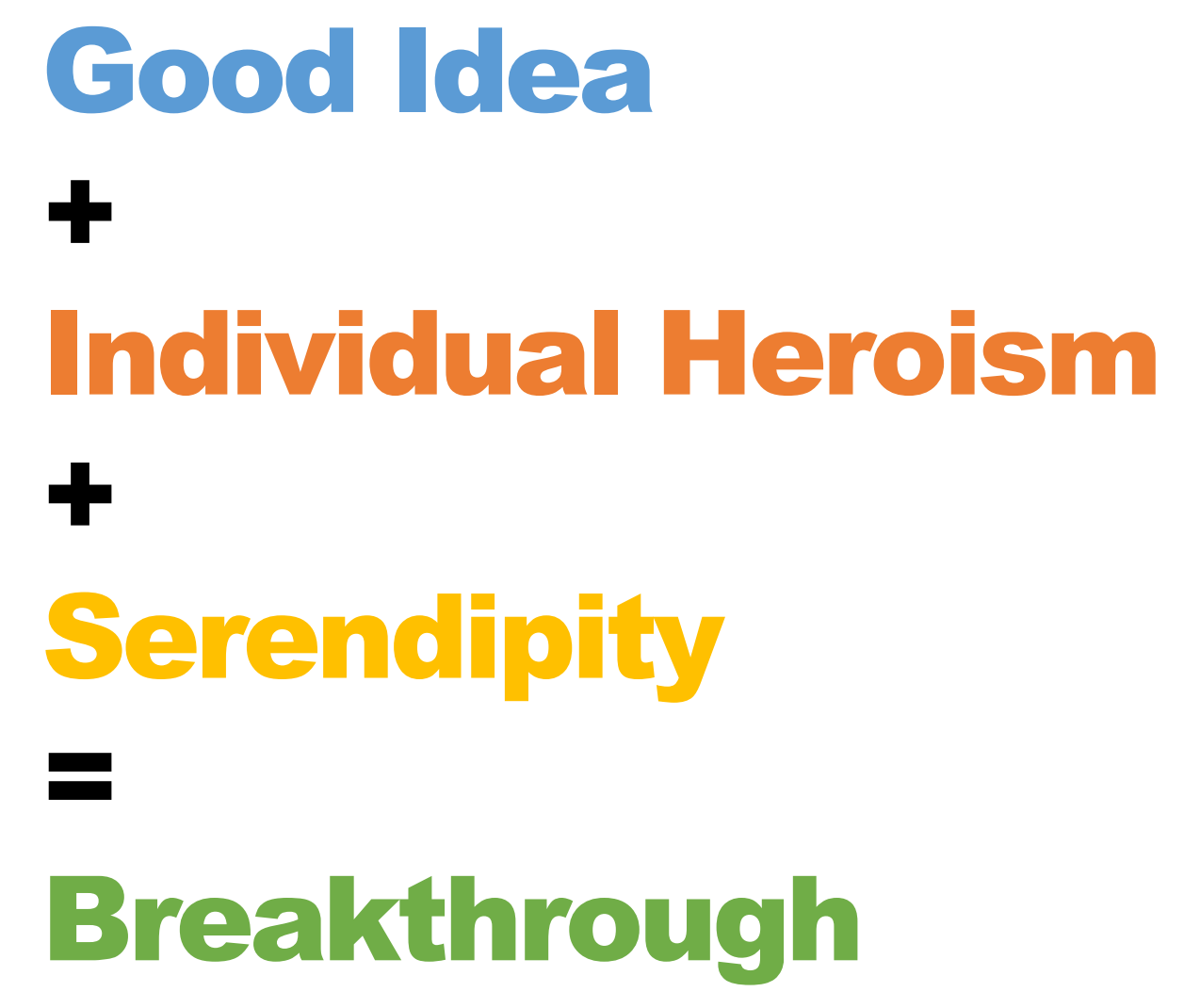


Extremes on the innovation spectrum

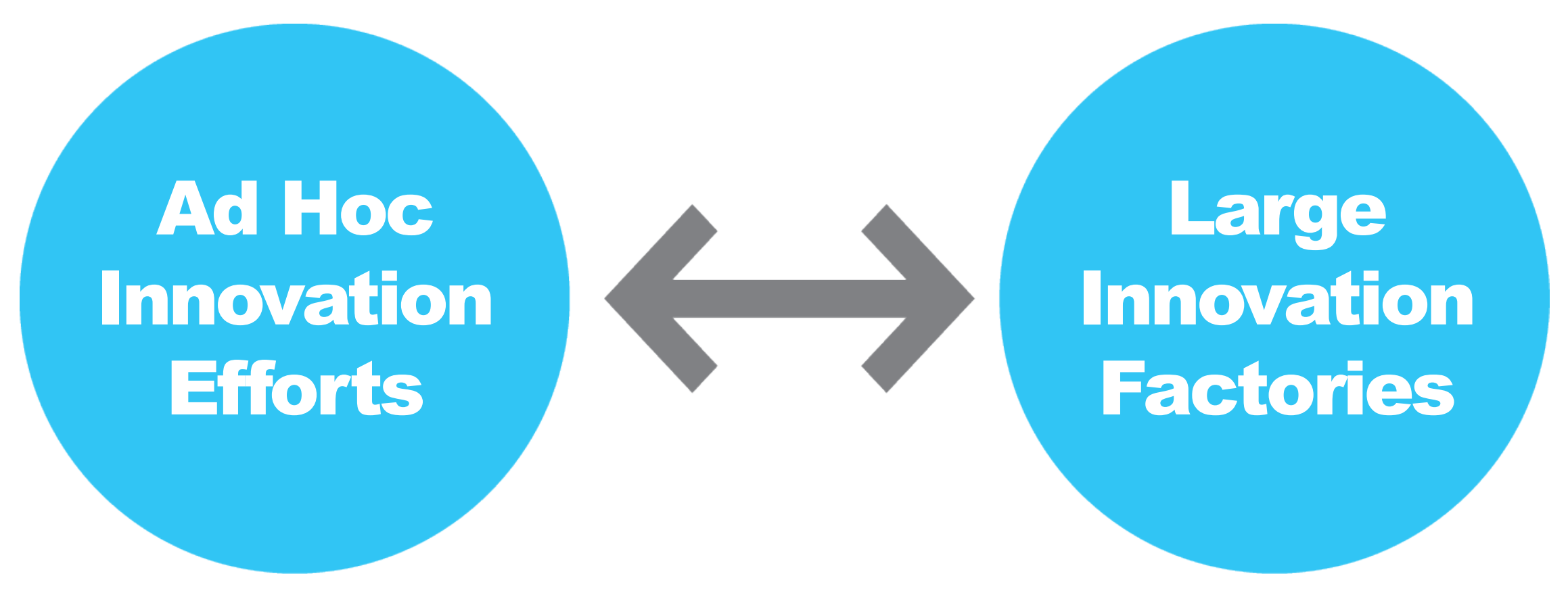


Regional innovation ecosystems

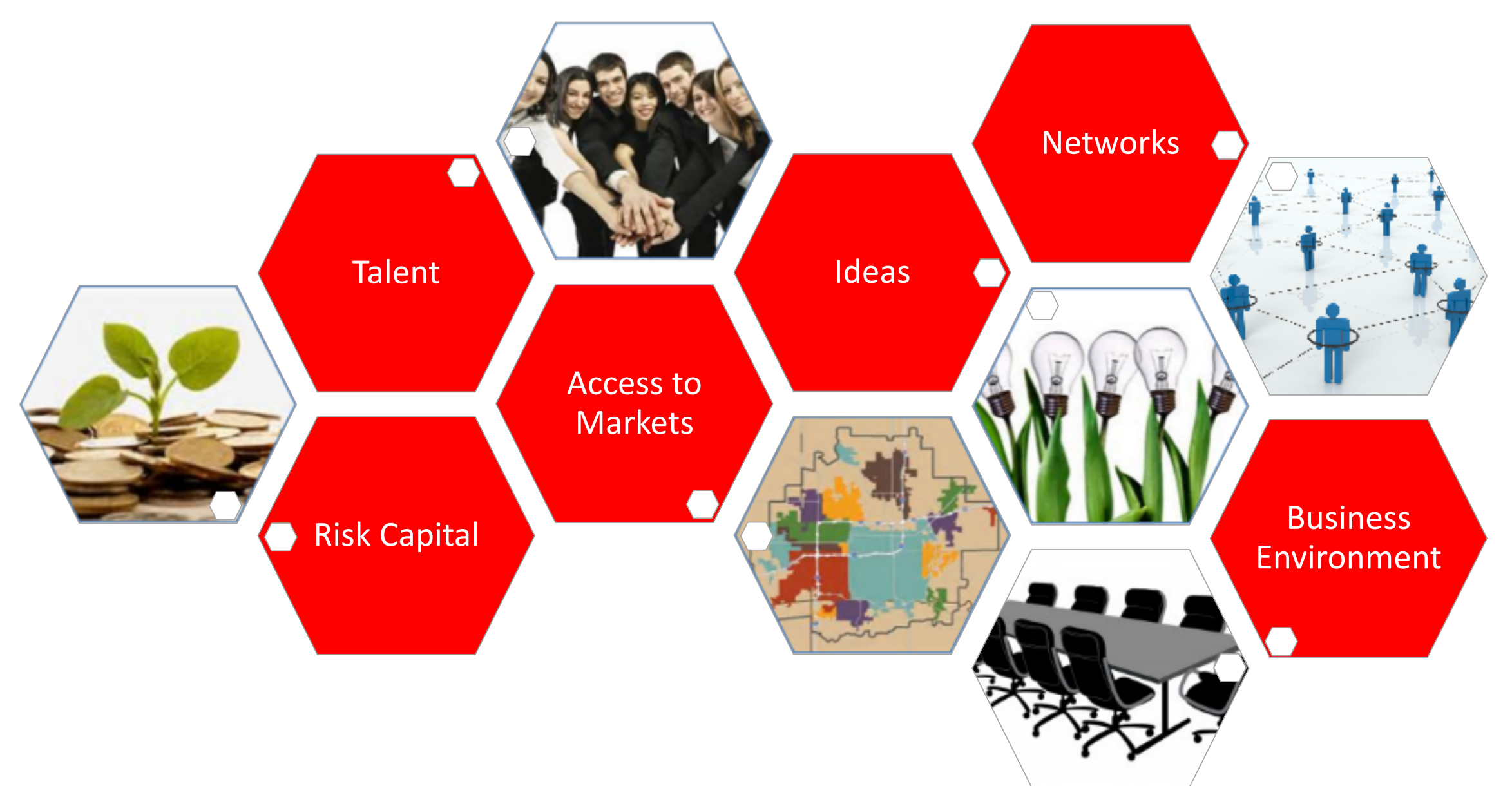




\section{Business environment}

- Relationships

- Trust

- Introductions

- CDAs

- Legal agreements

- Entrepreneurial culture

- Failure

- Regional industry expertise, focus

- 'Politics' of the ecosystem

\author{
If you want to go \\ fast, go alone. \\ If you want to go \\ far, go with others.
}

African proverb 


\section{Ecosystem stakeholders}

- Connectors

- Entrepreneurs

- Industry executives

- Economic developers

- Angel investors

- Venture capitalists

- Corporations

- Government

- Advocacy/membership organizations

- Professional services providers

- Accelerators

- Incubators

- Others

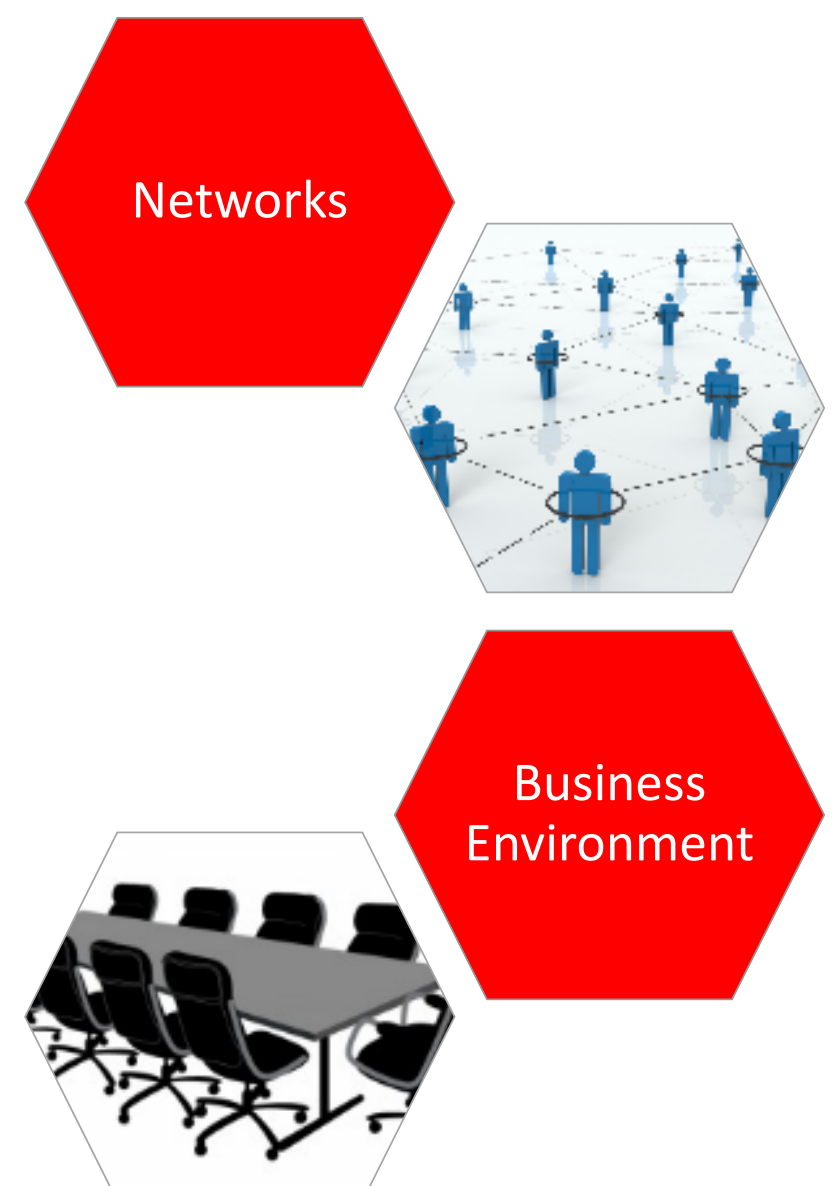




\section{Ecosystem connectivity}

- Connectors

- Entrepreneurs

- Industry executives

- Economic developers

- Angel investors

- Venture capitalists

- Corporations

- Government

- Advocacy/membership organizations

- Professional services providers

- Accelerators

- Incubators 


\section{Connectors}

- Who are they

- Why do they do it

- Are you one

- Should you be 


\section{Entrepreneurs}

- First time vs. serial

- Technician as entrepreneur

- Industry/sector expertise

- Technology expertise

- Current vs. deferred compensation requirements

- Build to sell vs. lifestyle 


\section{Industry executives}

- Licensee

- Research sponsor

- Would-be entrepreneur

- Would-be investor

- Would-be partner

- Would-be acquirer 


\section{Economic developers}

- Job creation

- Training programs

- Seed capital for programs

- Seed capital for startups 


\section{Angel investors}

- Source of capital

- 'Smart' money

- Experience

- Relationships

- Formal angel groups

- Triage

- [Interim] management 


\section{Venture capital}

- Team

- Market

- Technology

- Investment thesis

- Relationship 


\section{Corporations}

- Licensee

- Research sponsor

- Joint development

- Student employment

- Corporate venture 


\section{Government}

- $\quad$ Funding

- Training

- iCorps

- Regulations

- advocacy 
Advocacy / membership organizations

- Networking

- Information

- Promotion

- Advocacy 


\section{Professional service providers}

- In-kind support

- Network

- Diligence/triage 
Accelerators

- Training

- Mentorship

- Capital

- Resources 


\section{Incubators}

- Space

- Affordable 


\section{Your programs}

What is the purpose

- Engagement

- Ecosystem development

- Growth

- Filling gaps

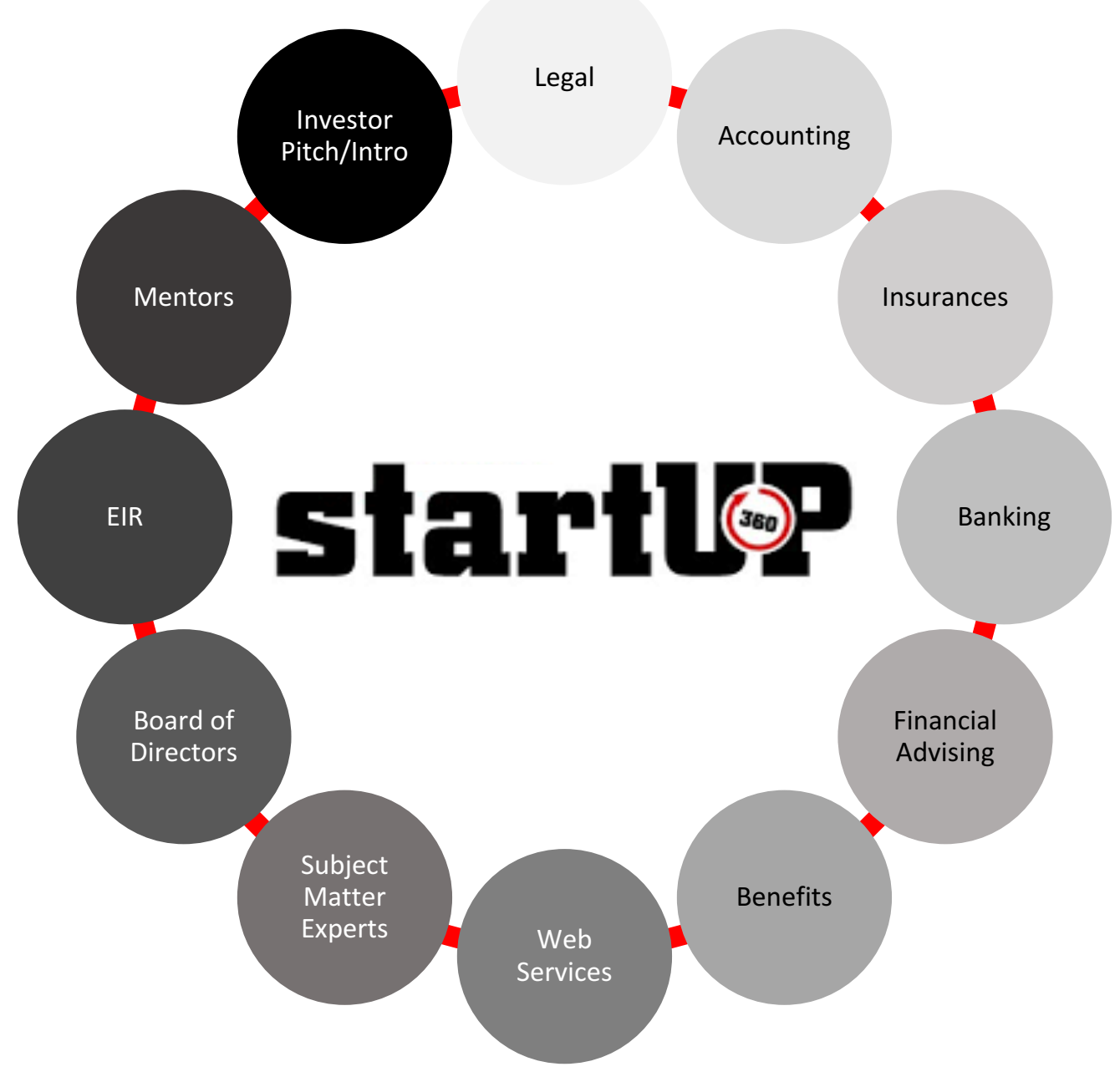

